\title{
Classificação da aptidão cardiorrespiratória de crianças de 5 a 10 anos, através de valores normativos no desempenho do teste yo-yo intermitente nível 1
}

Classification of cardiorespiratory fitness of children aged 5 to 10 years, using normative values in the performance of the intermittent level 1 yo-yo test

Clasificación de la aptitud cardiorrespiratoria en niños de 5 a 10 años, mediante valores normativos en la realización de la prueba intermitente de yo-yo 1

\section{Resumo}

Objetivo: propor padrões normativos de classificação da AC através do desempenho no teste Yo-Yo intermitente nível 1 (Yo-Yo) em crianças de 5 a 10 anos de idade. Materiais e métodos: o estudo foi composto por 1.060 crianças de ambos os sexos, para caracterização foi realizado avaliação antropométrica e utilizou-se o Yo-Yo para avaliação da AC. Resultados: as meninas apresentaram maior massa corporal $(\mathrm{p}<0.005)$ e menor AC em comparação os meninos ( $\mathrm{p}<0.001)$, os valores dos quatis com base no despenho do Yo-Yo foram distribuídos em baixo, moderado, alto e muito alto. Conclusão: o presente estudo apresentou uma proposta de valores de referência da AC, a utilização dos valores propostos pelo presente estudo, podem ser um instrumento fundamental no processo de avaliação da AC em crianças por parte de profissionais de saúde e professores.

Palavras-chave: Crianças; Aptidão cardiorrespiratória; Yo-Yo test. 


\begin{abstract}
Objective: to propose normative standards for the classification of CA through the performance in the intermittent YoYo test level 1 (Yo-Yo) in children from 5 to 10 years old. Materials and methods: the study consisted of 1,060 children of both sexes. Anthropometric evaluation was performed for characterization and the Yo-Yo was used to assess CA. Results: girls had higher body mass $(\mathrm{p}<0.005)$ and lower WC compared to boys $(\mathrm{p}<0.001)$, coatis values based on Yo-Yo performance were distributed in low, moderate, high and very high. Conclusion: the present study presented a proposal for reference values for CA, the use of the values proposed by this study, can be a fundamental instrument in the process of evaluating CA in children by health professionals and teachers.
\end{abstract}

Keywords: Children; Cardiorespiratory fitness; Yo-Yo test.

\title{
Resumen
}

Objetivo: proponer estándares normativos para la clasificación de CA a través del desempeño en el test de Yo-Yo intermitente nivel 1 (Yo-Yo) en niños de 5 a 10 años. Materiales y métodos: el estudio consistió en 1.060 niños de ambos sexos, se realizó una evaluación antropométrica para la caracterización y se utilizó el YoYo para evaluar la CA. Resultados: las niñas presentaron mayor masa corporal ( $p<0.005)$ y menor CC en comparación con los niños ( $p$ $<0.001$ ), los valores de coatis basados en el rendimiento del YoYo se distribuyeron en bajo, moderado, alto y muy alto. Conclusión: el presente estudio presentó una propuesta de valores de referencia para CA, el uso de los valores propuestos por este estudio, puede ser un instrumento fundamental en el proceso de evaluación de CA en niños por parte de profesionales de la salud y docentes.

Palabras clave: Niños; Aptitud cardiorrespiratoria; Prueba de yo-yo.

\section{Introdução}

A aptidão cardiorrespiratória (AC) é uma capacidade em constante alteração ao longo do desenvolvimento humano, tais alterações sofrem interferência de fatores associados ao estilo de vida. Dentre inúmeros fatores a elencar, destaca-se a faixa etária, sexo, nível de atividade da vida diária, prática de esportes, prática de exercícios físicos e a inatividade física (Ferrari et al., 2015). Portanto, compreender as diferentes atuações dos fatores supracitados, pode ser fundamental para explicar, em partes, as alterações da AC, uma vez que essa variável demonstra-se como um marcador de saúde essencial em crianças e adolescentes (Raghuveer et al., 2020). O cenário de avaliação desse importante marcador se demonstra importante para a saúde, pois, o baixo desempenho da AC estão associados ao surgimento de risco de doenças cardiovasculares e alterações metabólicas ao longo do desenvolvimento (Lang et al., 2018).

Nas últimas décadas, a AC apresentou um comportamento preocupante na população pediátrica brasileira, a qual veem apresentando AC abaixo do recomendado para a idade e para o sexo, com decréscimos em aproximadamente $60 \%$ de escolares de 7 a 12 anos de ambos os sexos (Costanzi et al., 2009)(Soares et al., 2014a). Condição favorável a fornecer menores oportunidades de desenvolvimento físico, motor e cognitivo a este público. (Batista et al., 2017; Ferrari et al., 2015; Soares et al., 2014b).

Em uma revisão sistemática, (Lang et al., 2018) observaram estudos clínicos com 319 mil crianças e adolescentes com faixa etária entre 5 a 17 anos de 32 países. Seus resultados demonstraram uma associação entre maior desempenho da AC e melhores indicadores de saúde como: menor adiposidade, ajuste dos biomarcadores cardiometabólicos, maior cognição, saúde mental, saúde psicossocial, autoestima, qualidade de vida, saúde óssea, musculoesquelética, desenvolvimento de habilidades motoras e menores lesões e / ou danos (Lang et al., 2018).

Diante do exposto, torna-se fundamental avaliar e acompanhar o desenvolvimento da AC de crianças e adolescentes tal qual sua prospecção em vários indicativos de saúde (Raghuveer et al., 2020). Além disso, é importante estabelecer padrões normativos para a AC para direcionar profissionais de saúde para os possíveis valores de referência para a aptidão cardiovascular. Esse monitoramento e acompanhamento, pode possibilitar a identificação de fatores de risco inerentes a desfechos cardiovasculares adversos e mortalidade no futuro. Sendo assim, tais padrões poderão ser utilizados como marcadores de saúde geral. 
Ainda há poucos estudos normativos para observar a AC na infância, principalmente a escolha de um teste que possua pontos de corte específicos por sexo e idade. Essa ausência de indicadores classificatórios e normativos aos testes que possuem uma representatividade de utilizá-los com baixo custo e de fácil aplicabilidade e reprodutibilidade faz com que alguns dos profissionais não os realizem. (Raghuveer et al., 2020). Assim, a utilização de um teste de campo se tornaria interessante desde que os participantes entendam as configurações e as regras do teste como forma de familiarização principalmente em idades menores (Schmitz et al., 2019). Para avaliar a AC os teste intermitentes se mostraram como uma ferramenta eficaz em crianças, conforme revisão sistemática com 7398 participantes com idades inferiores a 16 anos de idade(Schmitz et al., 2019) onde os autores concluem que os testes podem ser usados para avaliar e monitorar o desenvolvimento da aptidão relacionada a idade e sexo. Sabe-se ainda que o teste Yo-Yo intermitente nível 1 é amplamente utilizado como ferramenta de avaliação da AC.

Portanto o objetivo do estudo é propor padrões normativos de classificação da aptidão cardiorrespiratória por meio do desempenho obtido no teste Yo-Yo intermitente nível 1 em crianças de 5 a 10 anos de idade.

\section{Metodologia}

\section{Participantes}

Trata-se de uma pesquisa qualitativa do tipo observacional analítico do tipo transversal (Pereira et al., 2018).

Participaram 1.060 escolares de ambos os sexos, com idades entre 5 a 10 anos de idade, estudantes da rede de ensino pública e privada de Brasília e Regiões Administrativas, Distrito Federal. A seleção das crianças voluntárias a participarem do estudo, foi por método não probabilística do tipo intencional (Bhardwaj, 2019). Todos os participantes tiveram o termo de assentimento e consentimento livre esclarecido assinados pelos pais ou responsáveis. O estudo foi aprovado pelo Comitê de Ética e Pesquisa do Centro Universitário - UNIEURO ( $n^{\circ}$ 4.274.829). Os critérios de inclusão adotados foram a ausência de diagnóstico de patologias neurológicas e/ou psiquiátricas, não estar fazendo uso de medicamentos que alterassem os sentidos ou a cognição e não possuir nenhum impedimento físico ou auditivo para a realização dos testes. Todas as crianças possuíam aulas de Educação Física escolar pelo menos uma vez semanal. Para assegurar os critérios de inclusão do estudo, foi aplicado uma anamnese em forma de entrevista previamente a realização dos protocolos experimentais.

\section{Procedimentos}

A aplicação dos protocolos avaliativos foram feitas em apenas uma visita com a seguinte cronologia: Anamnese, avaliação antropométrica e o Yo-Yo test intermitente nível 1. Todos os avaliadores foram previamente treinados a aplicação dos testes escolhidos. A análise de dados foi realizada por pesquisadores diferentes dos que aplicaram os testes.

\section{Avaliação Antropométrica}

A avaliação antropométrica e composição corporal, consistiram na determinação da massa corporal (Balança, Welmy, 6155, Brasil), estatura (Estadiometro, Sanny, ES2040, Brasil), do índice de massa corporal $(\mathrm{IMC})=\frac{\text { Peso corporal }(\mathrm{kg})}{\text { Estatur } \mathrm{a}^{2}(\mathrm{~m})}$. Todas as avaliações antropométricas utilizaram-se os procedimentos descritos por Petroski et al (1995).

\section{Aptidão Cardiorrespiratória (Yo-Yo test intermitente nivel 1)}

Para avaliação da aptidão cardiorrespiratória utilizou-se o Yo-Yo test intermitente nível 1 (Yo-Yo IR1), que consiste em correr o máximo de tempo possível em percurso de ida e volta, considerando a distância de 16 metros para crianças com idade entre 6 a 9 anos (Ahler et al., 2012) e 20 metros para crianças acima de 9 anos de idade (Andersen et al., 2008). A 
velocidade foi controlada por estímulos sonoros e, quando a criança não atingia a zona demarcada em duas tentativas, era decretado o fim do teste. Todos os avaliadores eram aptos e treinados para aplicação do teste, todas as crianças foram acompanhadas por um adulto no decorrer do teste para auxilia-las no acompanhamento das velocidades determinadas pelos apitos sonoros. O teste já foi utilizado anteriormente em crianças brasileiras como os estudos conduzido por (K. R. de O. Batista et al., 2018; Mazzoccante et al., 2019; Mazzoccante, Corrêa, et al., 2020; Mazzoccante, De Luca Corrêa, et al., 2020; Silveira-Rodrigues et al., 2018; Soares et al., 2014b)

\section{Análise estatística}

A normalidade e homogeneidade dos dados foram testadas com o teste de Kolmogorov-Smirnov e Levene, respectivamente. Os dados foram expressos por meio do Test-t em medidas descritivas: média e desvio padrão. Para apresentação dos dados, estes estão descritos em sua totalidade e subdivididos em dois grupos referentes aos sexos masculinos e femininos. As apresentações dos valores normativos foram aplicadas a estratificação em quartis, os quartis foram divididos para o sexo e para cada faixa etária entre as classificações baixo, moderado, alto e muito alto. Para realização dessa análise e comparação da aptidão cardiorrespiratória entre as faixas etárias utilizou-se o teste ANOVA one-way. Utilizou-se o software SPSS versão 23, onde foi adotado um valor de $p<0,05$ para identificar possíveis diferenças estatísticas.

\section{Resultados}

Apresenta-se os dados de caracterização da amostra dispostos por sexo, em idade, massa corporal, estatura, índice de massa corporal e aptidão cardiorrespiratória como descrito na Tabela 1.

Tabela 1 - Características antropométricas e aptidão cardiorrespiratória do grupo de crianças do sexo feminino e masculino.

\begin{tabular}{|c|c|c|c|c|c|c|}
\hline \multirow{2}{*}{$\begin{array}{c}\text { Grupos etários } \\
\text { Variáveis } \\
\end{array}$} & \multicolumn{2}{|c|}{5 e 6 anos } & \multicolumn{2}{|c|}{7 e 8 anos } & \multicolumn{2}{|c|}{9 e 10 anos } \\
\hline & Feminino & Masculino & Feminino & Masculino & Feminino & Masculino \\
\hline Idade (anos) & $5,97 \pm 0,17 * * \# \#$ & $5,95 \pm 0,22 * \cdots \#$ & $7,21 \pm 0,41^{* *} *$ & $7,11 \pm 0,32^{* *}$ & $9,56 \pm 0,5$ & $9,59 \pm 0,49$ \\
\hline Massa corporal (kg) & $21,3 \pm 3,74 * * \# \#$ & $24,02 \pm 24,36^{* *}$ & $27,9 \pm 20,79 * *$ & $28,81 \pm 24,28 * *$ & $33,71 \pm 7,56$ & $37,49 \pm 15,83$ \\
\hline Estatura (m) & $1,17 \pm 0,06 * * \# \#$ & $1,17 \pm 0,05 * \cdots \#$ & $1,26 \pm 0,07^{* *}$ & $1,28 \pm 0,06^{* *} \cdot$ & $1,41 \pm 0,07$ & $1,41 \pm 0,07$ \\
\hline $\begin{array}{l}\text { Índice de massa corporal } \\
\qquad(\mathrm{kg} \cdot \mathrm{m} 2)\end{array}$ & $15,57 \pm 1,83^{*} *$ & $15,45 \pm 2,44^{*}$ & $16,2 \pm 3,11$ & $15,71 \pm 3,47^{2}$ k & $16,81 \pm 3,12$ & $17,85 \pm 10,22$ \\
\hline Distância & $229,59 \pm 99,5$ & $335,89 \pm 199,18 \#$ & $254,5 \pm 144,59$ & $419,39 \pm 282,38^{*}$ & $259,86 \pm 148,31$ & $359,56 \pm 234,58$ \\
\hline
\end{tabular}

* p <0,05 em comparação a faixa etária de 09 e 10 anos de idade do mesmo sexo; ** p<0,01 em comparação a faixa etária de 09 e 10 anos de idade do mesmo sexo; \# p<0,05 em comparação a faixa etária de 07 e 08 anos de idade do mesmo sexo; \#\# p<0,01 em comparação a faixa etária de 07 e 08 anos de idade do mesmo sexo. Fonte: Autores. 
Tabela 2 - Valores médios e desvio-padrão de classificação da aptidão cardiorrespiratória do sexo feminino apresentada de acordo com grupos etários.

\begin{tabular}{|c|c|c|c|c|c|c|}
\hline \multirow[b]{2}{*}{$\begin{array}{l}\text { Grupos } \\
\text { etários }\end{array}$} & \multirow[b]{2}{*}{$\begin{array}{c}\text { Classificação da } \\
\text { aptidão } \\
\text { cardiorrespiratória }\end{array}$} & \multicolumn{5}{|c|}{ Feminino } \\
\hline & & Idade (anos) & $\begin{array}{c}\text { Massa } \\
\text { corporal } \\
(\mathbf{K g})\end{array}$ & Estatura (m) & $\begin{array}{c}\text { Índice de } \\
\text { massa } \\
\text { corporal } \\
(\mathrm{kg.m2})\end{array}$ & Distancia (m) \\
\hline \multirow{4}{*}{ 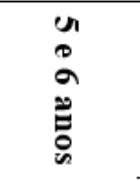 } & Baixo & $5,91 \pm 0,3$ & $22,08 \pm 4,86$ & $1,17 \pm 0,07$ & $15,9 \pm 2,36$ & $118,5 \pm 38,09 t$ \\
\hline & Moderado & $6 \pm 0$ & $21,03 \pm 3,64$ & $1,15 \pm 0,06$ & $15,77 \pm 1,79$ & $194,93 \pm 7,48 \mathrm{t}$ \\
\hline & Alto & $6 \pm 0$ & $20,34 \pm 2,03$ & $1,16 \pm 0,05$ & $15,22 \pm 1,1$ & $270,89 \pm 25,49 t$ \\
\hline & Muito alto & $6 \pm 0$ & $21,42 \pm 3,49$ & $1,18 \pm 0,05$ & $15,38 \pm 1,69$ & $356,33 \pm 40,65 \mathrm{t}$ \\
\hline \multirow{4}{*}{ 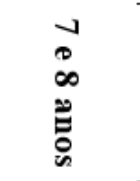 } & Baixo & $7,05 \pm 0,22 \mathrm{ab}$ & $31,48 \pm 35,67$ & $1,24 \pm 0,06$ & $16,15 \pm 3,94$ & $129,32 \pm 28,49 t$ \\
\hline & Moderado & $7,17 \pm 0,38 \mathrm{a}$ & $27 \pm 6,18$ & $1,26 \pm 0,06$ & $17 \pm 2,93$ & $205,17 \pm 14,92 \mathrm{t}$ \\
\hline & Alto & $7,28 \pm 0,45$ & $27,38 \pm 7,57$ & $1,28 \pm 0,08$ & $16,59 \pm 2,86$ & $266,21 \pm 21,16 \mathrm{t}$ \\
\hline & Muito alto & $7,4 \pm 0,49$ & $24,54 \pm 4,05$ & $1,26 \pm 0,07$ & $15,4 \pm 1,75$ & $450,4 \pm 162,46 \mathrm{t}$ \\
\hline \multirow{4}{*}{$\begin{array}{l}0 \\
0 \\
\stackrel{0}{0} \\
\vdots \\
0 \\
0\end{array}$} & Baixo & $9,73 \pm 0,45 b$ & $34,27 \pm 6,48$ & $1,4 \pm 0,06$ & $17,42 \pm 2,55 b$ & $130 \pm 31,91 \mathrm{t}$ \\
\hline & Moderado & $9,53 \pm 0,51$ & $34,5 \pm 9,52$ & $1,41 \pm 0,08$ & $17,08 \pm 2,85$ & $201,18 \pm 4,85 a$ \\
\hline & Alto & $9,38 \pm 0,49$ & $31,83 \pm 8,16$ & $1,4 \pm 0,08$ & $16,13 \pm 3,1$ & $257,26 \pm 19,83$ \\
\hline & Muito alto & $9,57 \pm 0,5$ & $34,71 \pm 7,15$ & $1,43 \pm 0,07$ & $16,98 \pm 2,78$ & $449,36 \pm 175,74$ \\
\hline
\end{tabular}

${ }^{a} \mathrm{p}<0,01$ em comparação a classificação da aptidão cardiorrespiratória "muito alto" do mesmo grupo etário; ${ }^{\mathrm{b}} \mathrm{p}<0,05$ em comparação a classificação da aptidão cardiorrespiratória "alto" do mesmo grupo etário; c $\mathrm{p}<0,05$ em comparação a classificação da aptidão cardiorrespiratória "moderado" do mesmo grupo etário; " $\mathrm{p}<0,05$ em comparação a todos os níveis de classificação da aptidão cardiorrespiratória do mesmo grupo etário. Fonte: Autores.

Tabela 3 - Valores médios e desvio-padrão de classificação da aptidão cardiorrespiratória do sexo masculino apresentada de acordo com grupos etários.

\begin{tabular}{|c|c|c|c|c|c|c|}
\hline \multirow[b]{2}{*}{$\begin{array}{l}\text { Grupos } \\
\text { etários }\end{array}$} & \multirow[b]{2}{*}{$\begin{array}{c}\text { Classificação da } \\
\text { aptidão } \\
\text { cardiorrespiratória }\end{array}$} & \multicolumn{5}{|c|}{ Masculino } \\
\hline & & Idade (anos) & $\begin{array}{c}\text { Massa } \\
\text { corporal }(\mathrm{Kg})\end{array}$ & Estatura (m) & $\begin{array}{c}\text { Índice de massa } \\
\text { corporal } \\
\text { (kg.m2) } \\
\end{array}$ & Distância (m) \\
\hline \multirow{4}{*}{ 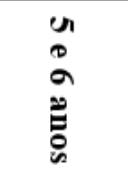 } & Baixo & $5,82 \pm 0,39$ & $22,69 \pm 4,37$ & $1,18 \pm 0,06$ & $16,12 \pm 2,23$ & $142,57 \pm 50,93 \mathrm{t}$ \\
\hline & Moderado & $6 \pm 0$ & $22,97 \pm 3,33$ & $1,18 \pm 0,05$ & $16,51 \pm 2,6$ & $246,63 \pm 14,1 \mathrm{t}$ \\
\hline & Alto & $6 \pm 0$ & $27,67 \pm 40,74$ & $1,17 \pm 0,05$ & $14,64 \pm 2,71$ & $383,53 \pm 70,86 \mathrm{t}$ \\
\hline & Muito alto & $6 \pm 0$ & $19,88 \pm 2,75$ & $1,15 \pm 0,03$ & $14,93 \pm 1,41$ & $684 \pm 156,37 \mathrm{t}$ \\
\hline \multirow{4}{*}{$\begin{array}{l}\checkmark \\
0 \\
\infty \\
ٍ ֶ \\
0\end{array}$} & Baixo & $7,14 \pm 0,35$ & $27,03 \pm 7,17$ & $1,28 \pm 0,06$ & $16,37 \pm 2,92$ & $166,8 \pm 28,26 \mathrm{t}$ \\
\hline & Moderado & $7,06 \pm 0,24$ & $31,65 \pm 33,52$ & $1,28 \pm 0,06$ & $16,11 \pm 3,4$ & $260,28 \pm 28,38 \mathrm{t}$ \\
\hline & Alto & $7,19 \pm 0,4$ & $26,93 \pm 6,18$ & $1,3 \pm 0,07$ & $15,87 \pm 2,71$ & $398,47 \pm 63,5 t$ \\
\hline & Muito alto & $7,06 \pm 0,24$ & $29,88 \pm 34,13$ & $1,26 \pm 0,06$ & $15,55 \pm 2,6$ & $834,98 \pm 213,43 t$ \\
\hline \multirow{4}{*}{$\begin{array}{l}0 \\
0 \\
ت \\
0 \\
00 \\
0\end{array}$} & Baixo & $9,63 \pm 0,49$ & $39,82 \pm 11,37$ & $1,42 \pm 0,07$ & $19,63 \pm 4,34$ & $192,82 \pm 44,86 \mathrm{t}$ \\
\hline & Moderado & $9,48 \pm 0,51$ & $35,67 \pm 10,18$ & $1,41 \pm 0,1$ & $17,64 \pm 2,97$ & $275,48 \pm 9,39 a$ \\
\hline & Alto & $9,61 \pm 0,49$ & $35,58 \pm 8,85$ & $1,42 \pm 0,06$ & $17,63 \pm 3,73$ & $346,48 \pm 34,81 \mathrm{a}$ \\
\hline & Muito alto & $9,59 \pm 0,5$ & $36,65 \pm 26,93$ & $1,41 \pm 0,06$ & $19,04 \pm 18,77$ & $672,55 \pm 258,27$ \\
\hline
\end{tabular}

a $p<0,01$ em comparação a classificação da aptidão cardiorrespiratória "muito alto" do mesmo grupo etário; ' $\mathrm{p}<0,05$ em comparação a todos os níveis de classificação da aptidão cardiorrespiratória do mesmo grupo etário. Fonte: Autores. 
Tabela 4 - Proposta de tabela de estadiamento para a Aptidão Cardiorrespiratória por meio do teste Yo-Yo Intermitente nível 1, apresentado pelos valores absolutos em metros dos grupos dos sexos e das faixas etárias.

\begin{tabular}{|c|c|c|c|c|}
\hline \multicolumn{2}{|r|}{ Variáveis } & \multicolumn{3}{|c|}{ Faixa etária } \\
\hline Sexo & Desempenho no teste Yo-Yo (m) & $5-6(n=193)$ & $7-8(n=443)$ & $9-10(n=424)$ \\
\hline \multirow{4}{*}{ 䭬 } & Baixo & $<160$ & $<160$ & $<160$ \\
\hline & Moderado & $160-223$ & $160-239$ & $160-239$ \\
\hline & Alto & $224-319$ & $240-303$ & $240-300$ \\
\hline & Muito alto & $>319$ & $>303$ & $>300$ \\
\hline \multirow{4}{*}{ 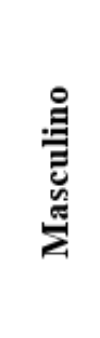 } & Baixo & $<200$ & $<202$ & $<240$ \\
\hline & Moderado & $200-279$ & $202-319$ & $240-299$ \\
\hline & Alto & $280-480$ & $320-572$ & $300-400$ \\
\hline & Muito alto & $>480$ & $>542$ & $>400$ \\
\hline \multirow{4}{*}{ సี } & Baixo & $<160$ & $<192$ & $<200$ \\
\hline & Moderado & $160-255$ & $192-255$ & $200-259$ \\
\hline & Alto & $256-352$ & $256-382$ & $260-360$ \\
\hline & Muito alto & $>352$ & $>382$ & $>360$ \\
\hline
\end{tabular}

Fonte: Autores.

Ao comparar as faixas etárias não se observou diferença entre as crianças em suas respectivas faixas etárias considerando os valores de aptidão cardiorrespiratória, descrito na Figura 1. 
Figura 1 - desempenho da aptidão cardiorrespiratória do sexo feminino (a) e masculino (b) de forma separada.

A) Sexo feminino

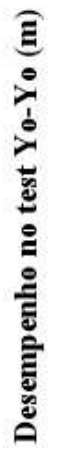

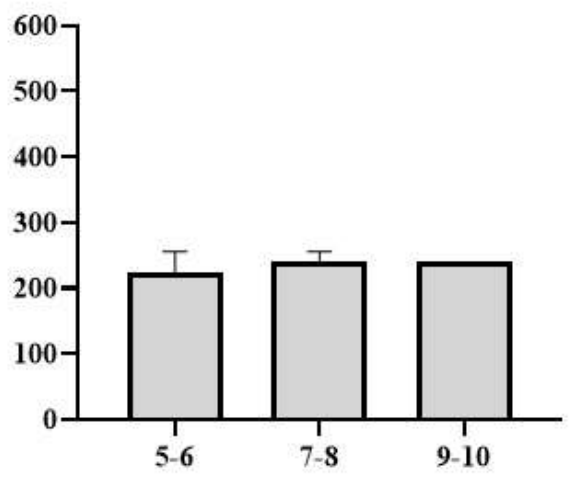

Faixa etária (anos)
B) Sexo masculino

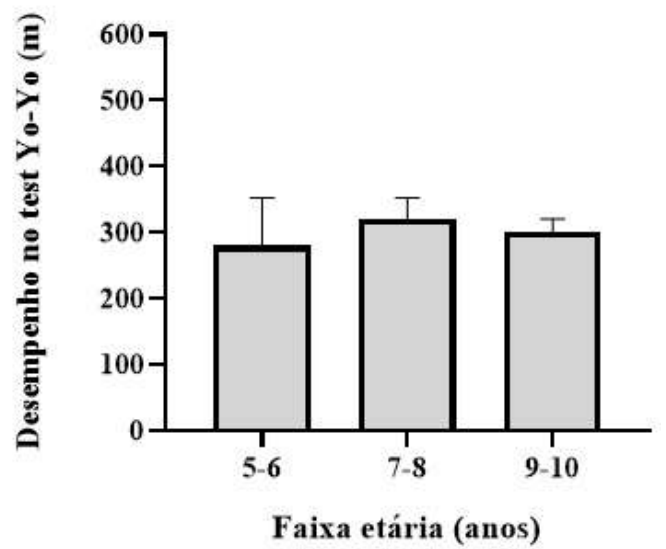

Fonte: Autores.

\section{Discussão}

O presente estudo sugeriu possíveis pontos de cortes para classificação da AC em crianças de ambos os sexos com idade de 5 a 10 anos por meio do teste Yo-Yo intermitente nível 1 que é um teste já utilizado em crianças brasileiras (Batista et al., 2018; Mazzoccante, De Luca Corrêa, et al., 2020; Mazzoccante \& Ribeiro, 2020; Penha et al., 2019) (Tabela 2). Além disso observou-se que as crianças não apresentam diferenças no desempenho da $\mathrm{AC}$ dos 5 até os 10 anos de idade.

Em recente declaração cientifica a American Heart Association, evidenciou acerca da importância em se avaliar e mensurar a $\mathrm{AC}$ em condições clínicas ou não, nesse mesmo estudo, considerou-se a $\mathrm{AC}$ como biomarcador importante para prever mortalidade pois, na pratica clínica, a AC em baixo nível está associada a um risco elevado de se desenvolver doença cardiovascular e ocasionar morte por todas as causas. (Ross et al., 2016).

Pereira et al (2008)(Pereira et al., 2008)(Pereira et al., 2008)(Pereira et al., 2008) observaram que o teste Yo-Yo intermitente nível 1 e o teste de cooper 12 minutos apresentam valores da potência aeróbia máxima correlata. O teste Yo-Yo intermitente nível 1 também é um teste já utilizado no público de crianças brasileiras como ferramenta de avaliação da potência aeróbia (Mazzoccante, De Luca Corrêa, et al., 2020) e se associa com melhores resultados de atenção, flexibilidade cognitiva e velocidade de processamento, que é explicado em parte devido a uma maior vascularização no número de transmissores e de regiões sinápticas (Mazzoccante \& Ribeiro, 2020).

$\mathrm{Na}$ literatura ainda é escasso a utilização do teste Yo-Yo intermitente nível 1 como medida de avaliação da AC e valores de classificação para o publico infantil no Brasil, entretanto o teste demonstra ser de facil aplicação (Lang et al., 2018). Ademais, Raghuveer et al (2020) sugere que o desenvolvimento de um processo de avaliação da aptidão cardiorrespiratória de baixo custo e de fácil acesso nas configurações de campo, podem ter uma forte significância no que tange a identificação precoce de possíveis riscos em jovens.

De acordo com Penha et al (2019), as crianças que possuem maior gordura corporal possuem pior desempenho no teste Yo-Yo intermitente nível 1. Llorente e Cantarero (2012) evidenciaram em crianças de 7 a 12 anos de idade, saudáveis e com massa corporal eutrófica, classificadas com baixa AC, apresentaram distúrbios no perfil metabólico e inflamatório em detrimento as crianças com maior AC, demonstrando assim a importância da avaliação da AC, como parâmetro de saúde infantil. Corroborando com esses achados, (Raghuveer et al., 2020) destacam que crianças com baixo condicionamento tem 
maior probabilidade de desenvolver prematuramente doenças crônicas, como, insuficiência cardíaca, diabetes do tipo 2 e pressão alta, além de terem maior risco de mortalidade por doenças cardíacas quando adultas. A AC quando abaixo dos valores recomendados se relaciona com a prevalência de dislipidemia em escolares. (Reuter et al., 2019).

Além disso, crianças com uma classificação satisfatória da AC apresentam um melhor desempenho em atividades cognitivas. Assim a $\mathrm{AC}$ e volumes cerebrais específicos estão associados à saúde cognitiva no momento do teste, bem como desempenham um papel no desempenho cognitivo futuro (Chaddock et al., 2012).

Algumas limitações são dignas de nota, como por exemplo, não foram estratificados o grupo de crianças em detrimento das atividades ambientais executadas, como, Educação Física escolar e prática esportiva, visto que tais condições beneficiam a AC, não foi empregado nenhum teste laboratorial para verificar a confiabilidade e reprodutibilidade do YO-YO intermitente nível 1 em crianças de 5 a 10 anos de idade amostra específica, entretanto, o YO-YO intermitente nível 1apresenta uma forte correlação e já tem sido amplamente utilizado para avaliar a AC em crianças no Brasil. (Batista et al., 2018; Ferrari et al., 2015; Mazzoccante, De Luca Corrêa, et al., 2020; Mazzoccante \& Ribeiro, 2020; L. Pereira et al., 2008).

Em contrapartida, o presente estudo avança na fronteira do conhecimento acerca da proposta de classificação da aptidão cardiorrespiratória com um número de crianças avaliadas bem expressivo. Além disso, o presente estudo esclarece e sugere possíveis pontos de classificação da AC como parâmetros normativos em discriminar a condição cardiorrespiratória de crianças, propiciando maiores elementos ao conhecimento acerca da $\mathrm{AC}$ de crianças em especial evidenciando o uso de uma ferramenta de fácil acesso e de baixo custo, dessa forma se tornando viável a sua reprodutibilidade por outros profissionais.

\section{Conclusão}

O presente estudo apresentou uma proposta de valores de referência da AC por meio do teste YO-YO intermitente nível 1. Entende-se ainda que a utilização dos valores propostos pelo presente estudo, podem ser um instrumento fundamental no processo de avaliação da $\mathrm{AC}$ em crianças brasileiras por parte de profissionais de saúde e professores. Especialmente por ser de fácil aplicação, compreensão e baixo custo, contribuindo para uma possível definição dos valores de normalidade, além de auxiliar estudos futuros por apresentar pontos de corte específicos em diferentes faixas etárias para AC em crianças brasileiras.

\section{Referências}

Ahler, T., Bendiksen, M., Krustrup, P., Wedderkopp, N., \& George, K. P. (2012). Aerobic fitness testing in 6- to 9-year-old children: Reliability and validity of a modified Yo-Yo IR1 test and the Andersen test. European Journal of Applied Physiology, 112(3), 871-876. https://doi.org/10.1007/s00421-011-2039-4

Andersen, L. B., Andersen, T. E., Andersen, E., \& Anderssen, S. A. (2008). An intermittent running test to estimate maximal oxygen uptake: The Andersen test. Journal of Sports Medicine and Physical Fitness, 48(4), 434-437.

Batista, K. R. de O., de Oliveira, M. G. D., Lemos, C. F. D. S., Couto, J. de O., Soares, N. M. M., \& Silva, R. J. D. S. (2018). Cardiorespiratory fitness in children and adolescents. Revista Brasileira de Cineantropometria e Desempenho Humano, 20(6), 535-543. https://doi.org/10.5007/19800037.2018v20n6p535

Batista, M. B., Romanzini, C. L. P., Castro-Piñero, J., \& Vaz Ronque, E. R. (2017). Validity of field tests to estimate cardiorespiratory fitness in children and adolescents: A systematic review. In Revista Paulista de Pediatria. 35(2), 222-233. Sao Paulo Pediatric Society. https://doi.org/10.1590/1984$0462 / ; 2017 ; 35 ; 2 ; 00002$

Bhardwaj, P. (2019). Types of sampling in research. Journal of the Practice of Cardiovascular Sciences, 5(3), 157. https://doi.org/10.4103/jpcs.jpcs_62_19

Chaddock, L., Hillman, C. H., Pontifex, M. B., Johnson, C. R., Raine, L. B., \& Kramer, A. F. (2012). Childhood aerobic fitness predicts cognitive performance one year later. Journal of Sports Sciences, 30(5), 421-430. https://doi.org/10.1080/02640414.2011.647706

Costanzi, C. B., Halpern, R., Rech, R. R., Bergmann, M. L. D. A., Alli, L. R., \& De Mattos, A. P. (2009). Associated factors in high blood pressure among schoolchildren in a middle size city, southern Brazil. Jornal de Pediatria, 85(4), 335-340. https://doi.org/10.2223/JPED.1913

Ferrari, G. L. D. M., Matsudo, V. K. R., \& Fisberg, M. (2015). Changes in physical fitness and nutritional status of schoolchildren in a period of 30 years (1980-2010). Revista Paulista de Pediatria, 33(4), 415-422. https://doi.org/10.1016/j.rppede.2015.03.001 
Lang, J. J., Belanger, K., Poitras, V., Janssen, I., Tomkinson, G. R., \& Tremblay, M. S. (2018). Systematic review of the relationship between $20 \mathrm{~m}$ shuttle run performance and health indicators among children and youth. In Journal of Science and Medicine in Sport. 21(4), 383-397. Elsevier Ltd. https://doi.org/10.1016/j.jsams.2017.08.002

Llorente-Cantarero, F. J., Pérez-Navero, J. L., De Dios Benitez-Sillero, J., Muñoz-Villanueva, M. C., Guillén-Del Castillo, M., \& Gil-Campos, M. (2012). Non-traditional markers of metabolic risk in prepubertal children with different levels of cardiorespiratory fitness. Public Health Nutrition, 15(10), 1827-1834. https://doi.org/10.1017/S1368980011003533

Mazzoccante, R. P., Corrêa, H. D. E. L., Santana, F. S. D. E., Câmara, M. A., Raquel, B., Sousa, C. D. E., Raquel, I., Sousa, C. D. E., Ferreira, A. P., \& Melo, G. F. D. E. (2020). Attention And Executive Function Are Predicted By Anthropometric Indicators, Strength, Motor Performance, And Aerobic Fitness In Children AGED 6 - 10 YEARS. Human Movement, 21(1), 40-48.

Mazzoccante, R. P., Corrêa, H. D. L., Queiroz, J. L. De, Sousa, R. C. De, Raquel, I., Sousa, C. De, \& Barboza, M. A. (2019). ARTIGO ORIGINAL A relação da prática esportiva com o desempenho motor, atenção seletiva, flexibilidade cognitiva e velocidade de processamento em crianças de 7 a 10 anos. 29(3), $365-372$.

Mazzoccante, R. P., De Luca Corrêa, H., Castro de Sousa, B. R., Castro de Sousa, I. R., Barboza Santos, M. A., \& Pimentel Ferreira, A. (2020). Aptidão cardiovascular e coordenação motora apresentam relação com a atenção, flexibilidade cognitiva e velocidade de processamento em crianças de 6 à 10 anos de idade? Educación Física y Ciencia, 22(1), e117. https://doi.org/10.24215/23142561e117

Mazzoccante, R. P., \& Ribeiro, H. S. (2020). a Capacidade Preditiva De Indicadores De Composição Corporal, Aptidão Aeróbia E Coordenação Motora Sobre a Atenção E Função Executiva Em Crianças De 6 a 11 Anos. Revista Brasileira de Ciência e Movimento, 28(1), 60-68. https://doi.org/10.31501/rbcm.v28i1.10094

Penha, J. T. da, Gazolla, F. M., Carvalho, C. N. de M., Madeira, I. R., Rodrigues-Júnior, F., Machado, E. de A., Sicuro, F. L., Farinatti, P., Bouskela, E., \& Collett-Solberg, P. F. (2019). Physical fitness and activity, metabolic profile, adipokines and endothelial function in children. Jornal de Pediatria, 95(5), 531537. https://doi.org/10.1016/j.jped.2018.04.010

Pereira, A. S., Shitsuka, R., \& Computação, L. E. M. (n.d.). Metodologia da pesquisa científica.

Pereira, L., Monteiro, A., Franca, J. E., Barreto, J., Pereira, R., \& Machado, M. (2008). Correlation among the stimate VO2max for Cooper 12 minutes test and for YoYo Endurance Test L1 in soccer players. The Brazilian Journal of Soccer Science, 01(1), 33-41.

Petroski, E. E. L., Pires-Neto, C. S., \& Neto, C. P. (1995). Validação de Equações Antropométricas para estimativa da densidade Corporal em Mulheres. In Revista Brasileira de Atividade Física e Saúde (Vol. 2, pp. 65-73).

Raghuveer, G., Hartz, J., Lubans, D. R., Takken, T., Wiltz, J. L., Mietus-Snyder, M., Perak, A. M., Baker-Smith, C., Pietris, N., \& Edwards, N. M. (2020). Cardiorespiratory Fitness in Youth: An Important Marker of Health: A Scientific Statement From the American Heart Association. Circulation, 1-18. https://doi.org/10.1161/cir.0000000000000866

Reuter, C. P., Brand, C., da Silva, P. T., Reuter, É. M., Renner, J. D. P., Franke, S. I. R., de Mello, E. D., Burgos, L. T., Schneiders, L. de B., \& Burgos, M. S. (2019). Relationship between dyslipidemia, cultural factors, and cardiorespiratory fitness in schoolchildren. Arquivos Brasileiros de Cardiologia, 112(6), 729736. https://doi.org/10.5935/abc.20190068

Ross, R., Blair, S. N., Arena, R., Church, T. S., Després, J. P., Franklin, B. A., Haskell, W. L., Kaminsky, L. A., Levine, B. D., Lavie, C. J., Myers, J., Niebauer, J., Sallis, R., Sawada, S. S., Sui, X., \& Wisløff, U. (2016). Importance of Assessing Cardiorespiratory Fitness in Clinical Practice: A Case for Fitness as a Clinical Vital Sign: A Scientific Statement from the American Heart Association. In Circulation (Vol. 134, Issue 24). https://doi.org/10.1161/CIR.0000000000000461

Schmitz, B., Pfeifer, C., Kreitz, K., Borowski, M., Faldum, A., \& Brand, S. M. (2019). Normative Yo-Yo Intermittent Recovery Level 1 and Yo-Yo Intermittent Endurance Level 1 test values of boys aged 9-16 years. Journal of Science and Medicine in Sport, 22(9), 1030-1037. https://doi.org/10.1016/j.jsams.2019.05.016

Silveira-Rodrigues, J. G., Soares, G. A., Lamounier, J. A., Soares, D. D., Damasceno, V. de O., \& Gonçalves, R. (2018). Relationship between aerobic capacity with Birth Weight and breastfeeding patterns in children: A cross-sectional study. Revista de Nutricao, 31(5), 467-477. https://doi.org/10.1590/167898652018000500004

Soares, N. M. M., dos Santos Silva, R. J., de Melo, E. V., \& de Oliveira, A. C. C. (2014a). Infuência da maturação sexual na aptidão cardiorrespiratória em escolares. Revista Brasileira de Cineantropometria e Desempenho Humano, 16(2), 223-232. https://doi.org/10.5007/1980-0037.2014v16n2p223

Soares, N. M. M., dos Santos Silva, R. J., de Melo, E. V., \& de Oliveira, A. C. C. (2014b). Infuência da maturação sexual na aptidão cardiorrespiratória em escolares. Revista Brasileira de Cineantropometria e Desempenho Humano, 16(2), 223-232. https://doi.org/10.5007/1980-0037.2014v16n2p223 